\title{
Les frères Jérôme et Jean Tharaud : avant-gardistes de la Négritude ?
}

\author{
Cheikh M. Ndiaye, Union College, Etats-Unis
}

Vous, cher ami, vous portiez en vous même un de ces beaux trésors cachés. Personne, je crois, n'a pénétré comme vous dans la mentalité des Noirs de l'Afrique Occidentale. Du Niger à la Côte et du Sénégal à la Gambie, vous connaissez leurs grandes langues et beaucoup de leurs idiomes locaux; dans les villages perdus de la brousse ou de la forêt où vous avez passé des années et des années, vous avez vécu leur vie ; vous avez cheminé à cheval au milieu d'eux ; avec eux vous avez descendu les rivières larges de plusieurs kilomètres, ou circulé de marigot en marigot sur leurs longues pirogues ou dans votre canot à pétrole, écoutant leurs palabres à longueur de journée ; et quand ils sont venus se battre à nos côtés, vous avez suivi la fortune d'un de leurs bataillons. Vous étiez pour eux le Toubab, l'homme blanc, l'homme aux oreilles rouges, le personnage puissant vers lequel ils se tournent tout naturellement dès qu'ils sont en difficulté soit avec l'Administration, soit avec leurs voisins, voire avec leur famille, le Blanc qui exerce sur leur esprit un prestige quasi miraculeux, quand il est comme vous, ou notre cher Édouard Fouqué, votre compagnon de là-bas, compréhensif, juste et bienveillant [...]. Ce récit, nous l'avons imaginé en vous écoutant causer, et pour lui donner la vie, vous nous avez apporté les mille détails nécessaires, tantôt dans la langue des Ouolofs, tantôt dans celle des Mandingues, que vous parlez l'une et l'autre - avec la même aisance que le patois du Périgord (Randonnée de Samba Diouf 3-4).

Ce Vous ou le destinataire de ces mots est André Demaison, né en 1883 au sud-ouest de la France, à Bordeaux précisément. Demaison fit ses débuts chez Maurel \& Prom, maison de commerce bordelaise implantée à Saint-Louis et à Gorée au Sénégal en 1828. Plus tard, en 1914, il s'engagea volontairement dans l'infanterie coloniale avant d'être muté comme interprète auprès des tirailleurs sénégalais en 1915 pour ses connaissances des langues africaines. Quant aux coauteurs de ces mots qu'on vient de citer, ce sont les deux frères Jérôme et Jean Tharaud, nés respectivement en 1874 et en 1877 à Saint-Junien au centre-ouest de la France. Prix Goncourt en 1906, les deux frères deviennent membres de l'Académie française, Jérôme en 1939, Jean en 1946. «Ce récit » auquel ils font allusion est La randonnée de Samba Diouf, un roman de guerre qu'ils ont coécrit et publié en 1922 chez Plon à Paris. Cet essai porte sur ce roman sur lequel je suis tombé par hasard en France dans une petite librairie à Rennes en 2008. J'avoue que je n'en avais jamais entendu parler avant et le peu que je savais sur ses deux auteurs, Jérôme et Jean Tharaud, relevait de bribes d'histoires. Je voyais toujours leur nom sur la liste des anciens membres de 
l'Académie française. La matière dont ils se sont inspirés pour écrire leur roman de guerre provient, comme ils l'indiquent, des « mille détails nécessaires, tantôt dans la langue des Ouolofs, tantôt dans celle des Mandingues » de Demaison, Français bien connu dans le milieu colonial entre 1900 et 1950 en Afrique occidentale française.

Pour un habitué de la littérature coloniale et du discours colonial en particulier, le caractère singulier de La randonnée de Samba Diouf ne laisse pas indifférent. Certes, il y a eu d'autres Tharaud durant et après l'époque coloniale. On pense aux missionnaires français à la fin du dixneuvième et au début du vingtième siècles tels les révérends Père Henri Gravrand, père Léonce Crétois et l'abbé Père David Boilat. On pense aussi à d'autres chercheurs européens qui leur sont postérieurs tels Jean-Paul Sartre, Charles Becker, Jacques Chevrier, Lylian Kesteloot, Marguerite Dupire et, plus récemment, Éric Déroo. Autant de voix connues dans la littérature africaine francophone, toutes d'origine européenne; ces auteurs ont produit des récits de voyage, chroniques, œuvres ethnolinguistiques, documentaires, bribes historiques, essais mythologiques qui s'inscrivent dans la dynamique du contre-discours colonial. Chacun à sa façon, ils ont choisi de réécrire ou de reconstruire l'histoire des peuples africains. Les premiers intellectuels noirs francophones qui s’inspirent d'eux, furent les co-fondateurs de la Négritude dans les années 1930. Faut-il rappeler que ce terme Négritude fut inventé en 1934 par Aimé Césaire. Léopold S. Senghor en donna une définition officielle: l'ensemble des valeurs culturelles et spirituelles des civilisations d'Afrique noire. A l'instar de leurs prédécesseurs européens, Césaire, Senghor et Léon G. Damas, entre autres, s'engagèrent ainsi dans l'édification de l'histoire et des valeurs culturelles du monde noir. Le Sénégalais, le Martiniquais, et le Guyanais remirent en question le projet colonial, y compris son eurocentrisme, son discours paternaliste et ses clichés. L'auteur, cinéaste, chercheur en anthropologie et en histoire Éric Deroo, producteur de Force Noire (2007), un film documentaire sur les tirailleurs sénégalais, résumera plus tard les stéréotypes coloniaux dans un entretien avec Timothy Mirthil le 4 mai 2006 :

Cet ensemble d'images est véhiculé sans que les Français ne s'intéressent particulièrement aux colonies mais chacun de ces stéréotypes tient lieu de discours sur les colonies. Lors de l'exposition coloniale de 1931, les visiteurs viennent voir un Palais des rêves, une gigantesque foire à neuneu. Ils ne se préoccupent pas de savoir quel intérêt ont les colonies et s'intéressent encore moins aux personnes qui y habitent. Là, se constitue une culture coloniale en creux, presque négative. Chaque Français est alors persuadé de connaître ces endroits mystérieux car il a lu un article, vu une affiche ou un film. Ce rapport aux colonies est basé sur un discours simple et une idéologie très artificielle. 
Les auteurs de La randonnée de Samba Diouf s'inscrivent dans cette dynamique de contrediscours, même si, il faut le rappeler, la mémoire (Demaison) qui a engendré leur roman était au service du colon. La connaissance de l'être africain et de ses rapports avec son cosmos y est notoire, ce qui montre l'effort et la volonté des frères Tharaud de comprendre et d'expliquer les énigmes du monde africain. Sans aucun doute, le texte a une stature avant-gardiste vis-à-vis du mouvement de la Négritude. Il a été publié en 1922, presque une décennie avant la Négritude. Samba, le personnage central du roman, nous dit-on, est « un Diouf, fils de Diouf, du village de Niômi » (34). Le patronyme « Diouf » tout comme « Niômi », le nom de son village, campent à la fois l'appartenance généalogique et ethnique et l'origine du personnage. Samba est un Sérère (épelé Sereer aussi) originaire d'un village de pêcheurs au sud de Dakar. Les frères Tharaud le distingue à travers ces marques identitaires qu'ils garnissent avec plus de détails sur le mode de vie de son village :

Dans le pays des Niôminkas, au village de Niômi, à mi-chemin des grands fleuves qui coulent au sud du pays de Dakar, les Anciens causaient sous le m'bar, le hangar recouvert de paille dont le toit descend jusqu'à terre et où, dans la saison des pluies, les gens viennent passer les longues heures de la journée. Un petit feu qui brûlait au milieu l'emplissait de fumée. Dans un coin, le forgeron réparait des outils, et le bruit des soufflets en peau de chèvre, qu'un enfant gonflait et dégonflait tour à tour, accompagnait d'un rythme monotone les conversations paisibles (15).

Le sens de l'observation est remarquable dans cette description du « hangar » de Niômi faite par des auteurs présumés non avertis. Il est intéressant de noter le caractère analogique ou intertextuel entre le roman, cette description surtout, et L'enfant noir de Camara Laye publié plus tard en 1954. Nombreux sont d'ailleurs les éléments narratifs dans La randonnée de Samba Diouf qui rappellent au lecteur L'enfant noir. Dans son roman autobiographique, Laye reprend le cadre spatial (un village), l'univers du forgeron et la dimension sociale inhérente à son lieu de travail (atelier). La description que fait le jeune personnage de l'atelier de son père, nous rappelle à bien des égards le « hangar » de Niômi dans La randonnée de Samba Diouf. En effet Laye nous raconte :

Cet atelier était la maîtresse pièce de notre concession. Mon père s'y tenait généralement, dirigeant le travail, forgeant lui-même les pièces principales ou réparant les mécaniques délicates [...], j'ai pris l'habitude de m'accroupir dans l'atelier et de regarder briller le feu de la forge. (111)

De tous les travaux que mon père exécutait dans l'atelier, il n'y en avait point qui me passionnât davantage que celui de l'or ; il n'y en avait pas non plus de plus noble ni qui requît plus de doigté [...]. Le griot s'installait, préludait sur sa cora, 
qui est notre harpe, et commençait à chanter les louanges de mon père. Pour moi, ce chant était toujours un grand moment. J'entendais rappeler les hauts faits des ancêtres de mon père, et ces ancêtres eux-mêmes dans l'ordre du temps ; à mesure que les couplets se dévidaient, c'était comme un grand arbre généalogique qui se dressait, qui poussait ses branches ici et là, qui s'étalait avec ses cent rameaux et ramilles devant mon esprit. La harpe soutenait cette vaste nomenclature, la truffait et la coupait de notes tantôt sourdes, tantôt aigrelettes. Où le griot puisait-il ce savoir? Dans une mémoire particulièrement exercée assurément, particulièrement nourrie aussi par ses prédécesseurs, et qui est le fondement de notre tradition orale. (21-22)

L'enfant noir, rappelons-le, est bien postérieur au roman des Tharaud. La peinture que fait le jeune Laye de l'atelier de son père, forgeron lui aussi, nous plonge dans l'univers traditionnel africain. Le narrateur parle de « griot » (mémoire), de « chant » (musique), et surtout du rapport intrinsèque entre le « feu » et le travail de l'or dans la forge de son père. Autant La randonnée de Samba Diouf dépeint-elle «le m'bar» ou le hangar de Niômi comme un lieu symbolique en tant qu'espace social et source de création, autant L'enfant noir restitue-t-il toute une mémoire à travers la fonction didactique et sociale du griot et du forgeron dans l'atelier. L'allusion que font les Tharaud aux «Anciens » et à leurs « conversations paisibles » sous le «m’bar » fait non seulement écho aux traditions orales, elle évoque aussi une marque culturelle, celle de la gérontocratie. Le vieux Bakari en donne l'illustration : «Ce jour-là, les causeurs étaient nombreux. Il y avait le chef du village, le vieux Bakari Silla, d'un âge extraordinairement avancé, et qui plusieurs fois par jour répétait cette phrase : «En vérité, dans tout le pays, je puis lever le doigt et dire : 'Je suis l'aîné !' » (12). Le vieil homme revendique et déclame ainsi son statut social. « Son âge... avancé » qui lui confère la sagesse légitime son autorité (il est le chef du village) et son droit à la parole. Senghor évoquera plus tard cette vieillesse symbolique en Afrique noire en 1964 dans son poème «NDessé » où il supplie sa vieille mère de lui dire des contes et de lui raconter l'histoire de ses propres origines : «Mère, on m’écrit que tu blanchis comme la brousse à l'extrême hivernage... Ta saison belle avec sept fois neuf ans sans nuages... Mère parle-moi... Redis-moi les vieux contes des veillées noires, que je me perde par les roues sans mémoire... Dis-moi donc l'orgueil de mes pères ! »(Hosties noires, 81-82). Les frères Tharaud à travers le vieux Bakari et Senghor à travers sa vieille mère valident l'idée que, dans la société traditionnelle africaine, les Anciens, les Griots, et les Conteurs étaient la mémoire de leur peuple et des «Maîtres de la parole ». Mamadou Kouyaté en fait une illustration dans Soundjata ou l'épopée mandingue, roman épique de Djibril T. Niane publié en 1960 : 
Je suis griot. C'est moi Djeli Mamadou Kouyaté, fils de Bintou Kouyaté et de Djeli Kedian Kouyaté, maître dans l'art de parler. Depuis des temps immémoriaux, les Kouyaté sont au service des princes Kéita du Manding : nous sommes les sacs à parole, nous sommes les sacs qui renferment des secrets plusieurs fois séculaires. L'Art de parler n'a pas de secret pour nous ; sans nous les noms des rois tomberaient dans l'oubli, nous sommes la mémoire des hommes ; par la parole nous donnons vie aux faits et gestes des rois devant les jeunes générations. Je tiens ma science de mon père Djeli Kedian qui la tient aussi de son père ; l'Histoire n'a pas de mystère pour nous; nous enseignons au vulgaire ce que nous voulons bien lui enseigner, c'est nous qui détenons les clefs des douze portes du Manding ${ }^{1}(9)$.

Dans son article «La parole pensée, impératifs et contraintes dans la littérature orale », Papa M. Sène note « qu'il existe des textes oraux acceptés et reconnus par le corps social comme littéraires parce que différents de la banalité du discours quotidien » (5). Les Tharaud valident cette grille de lecture sur les textes oraux en l'appliquant au cadre spatial du «m'bar » dans La randonnée de Samba Diouf. Les figures et objets qui composent ce lieu tels les «Anciens », le « forgeron», le « petit feu », et les «causeries » sont symboliques ; ils revêtent une caractéristique orale et portent, chacun, une connotation assez savante. L'héritage qui sert de déclic au roman, nous plonge dans le système matrilinéaire traditionnel africain où le droit de succession revenait au neveu (pas au fils). La randonnée de Samba Diouf prend ainsi l'allure morphologique du conte, y compris toutes ses fonctions. Il y a une situation initiale d'équilibre jusqu'au jour où Samba quitte son village. Ce déclic engendré par la mort de Baba Dialo correspond à ce que Vladimir Propp appelle « motif de départ » dans la Morphologie du conte. Baba, frère de la mère de Samba, est mort dans le pays des Foulahs laissant à son neveu six vaches, des moutons et des chèvres pour héritage (25). Samba quitte ainsi Niômi à destination du pays des Foulahs. Si son chemin est parsemé d'épreuves morales et physiques, les Tharaud en font un prétexte littéraire pour étaler les us et coutumes des différents pays qu'il traverse. Samba parcourt des villages inconnus où il dort ou passe des journées. Aux formes de salutation littéralement traduites dans les langues du pays, le récit rend compte de certaines valeurs culturelles locales telles que l'hospitalité ou la téranga (mot oualof/wolof) dont profite l'hôte étranger. Partout où il passe, Samba est traité avec tous les égards. Riz, couscous et viande en abondance lui reviennent de droit. On parle même des baganes de vin de palme qui lui sont offerts ici et là. Le récit évolue ainsi de façon linéaire sans rebondissement majeur jusqu'au moment où Samba confronte un obstacle insurmontable : une conspiration. Parlant de leur victime, Farba Cissé, l'un des conspirateurs, se confie à son acolyte : «Ce Diouf est un de ces impies, tu l'as bien entendu, qui boivent jusqu'à perdre la tête et ne plus reconnaître 
leur père et leur mère. Envoie chercher chez Monna Badhji - un impie comme lui- une bagane de vin de palme et, lorsqu'il sera ivre, nous l'attacherons avec ces cordes et nous le conduirons au Manso » (81). Le Manso n'est autre que l'administrateur colonial français qui commandait la région (70). Ce complot musulman contre Samba, une sorte de digression, apparaît ainsi comme une rupture à la structure narrative. Les musulmans accusent Samba d'être un «impie » qu'il faut enivrer de «vin de palme » pour le prendre en otage et le délivrer au Manso. Ce dernier a ordonné à chaque village d'« amener un homme par cent habitants » (70) pour aller combattre les Allemands au nom de la France. Les frères Tharaud traitent ainsi l'Islam et la colonisation de forces de perturbation aux sociétés traditionnelles africaines. A la question «pourquoi nous ontils appelés pour faire la guerre avec eux ? (109), Amadou Lamine Cissé, le caporal, répond :

Tu es jeune, Arouna Dia, et tu n'es qu'un enfant. Et tu n'as pas connu le temps où les rois étaient les maîtres, et où tu ne savais jamais, le soir, en t'endormant dans ta case, si on ne te trouverait pas, le lendemain, avec la tête coupée, et si ta femme et tes enfants ne seraient pas emmenés en esclavage. Mais aujourd'hui tu peux t'endormir en paix, et tes enfants de vivre à l'endroit où ils sont nés. Tu peux porter les habits que tu veux, sans rendre jaloux les fils du roi. Si on te frappe, tu te plains au tribunal. Chacun peut avoir de l'honneur. Et lorsque tu voyages, tu trouves des puits sur ta route, et tu peux conduire ton troupeau d'une seule main. C'est pour cela que les Toubabs nous demandent de les aider maintenant. (109-110)

Amadou Lamine, l'auteur de cette réplique est un caporal, chargé de diriger le 113e bataillon des tirailleurs sénégalais. Le lecteur découvre qu'il « avait été l'élève des missionnaires de Mgr Jalabert » et qu' « il savait beaucoup de choses qu'on apprend dans les livres des Toubabs » (106107). Le mot Toubab en langue ouolof (wolof) désigne le Blanc ; c'est une appellation allusive au caractère mystérieux et étranger de l'homme blanc à l'époque coloniale. La réplique du caporal, plus qu'une défense de son rang privilégié, montre qu'il assume pleinement son statut de collaborateur colonial. En justifiant la campagne de recrutement de soldats africains par le commandant français, il défend et incarne l'ordre colonial. Cela laisse entrevoir une substitution de rôles ; le colonisé fait désormais le travail du colonisateur. Le caporal Cissé est interpellé par un autre tirailleur en ces termes :

Je ne veux rien t'apprendre caporal lui dit-il, à toi qui as mangé le pain des Toubabs, qui sais lire dans leurs livres et qui connais bien des choses qui nous sont cachées. Ta peau est noire comme la nôtre, mais tu t'approches du savoir des Toubabs. Je ne veux donc pas t'enseigner le chemin que tu dois suivre. Mais va trouver demain le Toubab capitaine, et dis-lui ce qui est dans nos esprits. En vérité la mort n'est rien. Si ce n'est pour aider les Toubabs à faire la guerre qu'on nous a conduits ici, qu'ils nous fassent lever ! Notre sabre est coupant et notre main est solide ! Je te le dis 
encore, et tu le sais, Lamine, la mort ne nous importe pas. Qu'on nous fasse livrer la bataille ! Celui qui mourra mourra, mais celui qui en échappera, qu'on le renvoie dans son pays. La guerre seule nous a amenés ici, et non pas le travail d'esclave que nous avons fait jusqu'à présent. (161-162)

Ce penchant belliqueux du tirailleur traduit un cri de révolte. Soldat et porte-parole de son groupe, le tirailleur dévoile leur « condition humaine » au sens existentialiste du terme. Aux conditions de vie - froid, pluies - s'ajoutent les corvées auxquelles ils sont assujettis. Les tirailleurs n'arrêtent pas de tirer des cailloux d'une carrière. Pire, le commandant ne leur explique pas le motif de cette corvée. Le lecteur découvrira plus tard que c'était pour les habituer au bruit des canons avant la descente sur le front. Les frères Tharaud entrent ainsi dans le psychique du tirailleur. C'est un soldat angoissé par la guerre, il s'adapte mal au froid occidental et sa présence au front lui est absurde. Sous l'emprise du signal à l'assaut, un tirailleur lâche ces mots : « Nous sommes dans la main de Dieu et des Toubabs et notre souffle n'a plus beaucoup de valeur » (178). Ce sentiment d'impuissance, mort symbolique de la masculinité, fait penser à « la crainte de la castration » chez Freud. En effet le tirailleur redoute son incapacité face au destin ; son expérience de la guerre est vécue comme une menace phallique. Sur ce, il bascule dans la résignation à la merci de « Dieu et des Toubabs ». Autant l'attente à la guerre est vécue dans l'angoisse, autant son spectacle est terrorisant. Samba Sarr surnommé le chamelier raconte à ses camarades restés dans une tranchée ce qu'il vient de voir sur le champ de bataille : «Sur le chemin que je suivais, mes yeux ont vu une chose qui a crevé mon cœur. Une balle de kanou a fait un trou plus large qu'un grenier d'arachides et déterré deux toubabs. Et si tu passais par-là, tu verrais des jambes et une tête qui n’ont pas de sépulture. Les bêtes viendraient les manger bientôt » (174). La force destructrice des armes symbolisée ici par le «kanou » (altération du mot français «canon ») sert de prétexte aux tirailleurs pour un débat métaphysique sur la mort. Le chamelier ne redoute pas la mort et préfêre tous les malheurs de la vie à celui d'être sans sépulture car, dit-il, dans son pays sérère, à Mbadane, « lorsqu'un homme était mort, on creusait un large trou profond où l'on enfouissait tout entière la case du défunt. Au milieu de sa case, lui-même était placé debout, soutenu par un piquet, avec son collier de perles rouges et ses armes, et des calebasses d'eau et de mil, et tout ce qui était nécessaire durant le long voyage qu'il allait faire dans l'autre vie. Ensuite on recouvrait le tout avec des arbres et de la terre » (175). Aux yeux de Samba Sarr, le lieu étranger de la guerre est une menace au code ancestral. Il craint que le propre rituel de la sépulture, une pratique du passage à l'autre vie, ne soit pas respecté. Les frères Tharaud restituent ainsi une croyance traditionnelle aux rituels qui 
doivent être associés à la mort pour la rendre possible. De tels rituels assurent la réincarnation dont Samba fait allusion et dont Senghor fera plus tard écho en 1947 dans sa préface aux Nouveaux contes d'Amadou Koumba de Birago Diop. Dans sa préface, Senghor témoigne qu' «En Afrique, le réel n'acquiert son épaisseur, ne devient vérité qu'en brisant les cadres rigides de la raison logicienne, qu'en s'élargissant aux dimensions extensibles du surréel ». Au fait, nombreux sont les rituels que les Sérères, groupe ethnique de Senghor, associent à la mort et qui font d'elle un passage vers l'au-delà. Ce déterminisme entre en jeu dans le discours des tirailleurs à travers le roman des Tharaud. Aux prières escomptées du griot dans le bataillon, un tirailleur répond : « Moi, j'ai quelque chose de meilleur que les prières de tes vieilles. Que j'en perde la vie ! mais j'ai payé d'une génisse et d'un mouton un téré qui me vient de Chir Bala M'Baki lui-même, à qui rien n'est caché de ce qui a été, de ce qui est et de ce qui sera »(192-193). Un autre tirailleur d'ajouter : «Moi, j'ai bien sur ma poitrine et à ma ceinture deux térés qui n'ont pas de semblables contre les balles et les sabres, et un troisième au bras contre les armes qui assomment » (193). Les tirailleurs se confient ainsi les uns aux autres. Leur discours sur le pouvoir occulte cache leur angoisse face à la guerre. Les térés dont ils parlent ne sont autres que des amulettes, des objets rituellement préparés par des shamans et des marabouts pour les protéger contre la mort. Le téré joue ainsi le rôle d' «adjuvant » comme dirait Propp dans La morphologie du conte. Cette amulette, espoir de (sur)vie, leur permet de vaincre la mort dans l'espoir de retourner au pays après la guerre. L'Allemand, aux yeux des tirailleurs et aux yeux des Français aussi, est l'incarnation du mal et seuls les térés lui sont invincibles. Les tirailleurs s'y accrochent et croient que leur vertu magique est à la hauteur de l'ennemi si elle ne lui est pas supérieure. Le téré, rituel de sépulture, tout comme d'ailleurs le masque africain, relèvent du surréel, ils « défient le Temps » ${ }^{2}$ comme dit Senghor. Ces croyances locales seront ultérieurement présentes dans la Négritude et dans la littérature africaine en général, y constituant même un socle thématique. Dans sa postface pour Ethiopiques, un recueil de poèmes publié en 1956, Senghor écrit :

Et puisqu'il faut m'expliquer sur mes poèmes, je confesserai encore que presque tous les êtres et choses qu'ils évoquent sont de mon canton : quelques villages sérères perdus parmi les tanns ${ }^{3}$, les bois, les bolongs ${ }^{4}$ et les champs. Il me suffit de les nommer pour revivre le Royaume d'enfance [...]. J'ai donc vécu en ce royaume, vu de mes yeux, de mes oreilles entendu les êtres fabuleux par-delà les choses : les Kouss ${ }^{5}$ dans les tamariniers, les Crocodiles, gardiens des fontaines, les Lamantins qui chantaient dans la rivière, les Morts du village et les Ancêtres, qui me parlaient, m’initiant aux vérités alternées de la nuit et du midi. (Euvre poétique, 160) 
En déclamant que « les Morts du village et les Ancêtres » lui «parlaient, [1]'initiant aux vérités alternées de la nuit et du midi », Senghor fait allusion au lien intrinsèque entre le réel (monde humain ou physique) et l'irréel (l'univers des ancêtres). Dans La civilisation sereer : Pangool, Henry Gravrand réitère ces croyances traditionnelles: "l'esprit sérère ressent de façon expérimentale l'existence d'une puissance qui le dépasse et d'où dépend son destin » (101). Chez les Sérères, la source de cette «puissance » transcendantale est Roog Seen, l'Être Suprême. Pour eux, les ancêtres sont les mediums entre Roog Seen (Dieu) et les êtres humains ; ils s'inclinent devant leurs Pangools (Esprits des ancêtres) pour recueillir la protection divine et pour éloigner ou exorciser le mal. Senghor déclame cette ascendance des ancêtres dans un de ces poèmes : «Et mon cœur de nouveau sur les marches de la haute demeure. Je m'allonge à terre à vos pieds, dans la poussière de mes respects $\mathrm{A}$ vos pieds ancêtres présents, qui dominez fiers la grande-salle de tous vos masques qui défient le Temps » (Chants d'ombre, «Le retour de l'enfant prodigue », poème IV). Ce retour prodigue à la « haute demeure » ou au pays des ancêtres révèle non seulement la dévotion du poète, elle témoigne aussi de son allégeance aux Pangools. Les frères Tharaud traitent aussi du thème du retour au plan séculaire. Quand Samba rentre au pays après la guerre, on organise une fête à son honneur. Les co-auteurs font tout un décor de la scène en exhibant des images et des couleurs locales assez pittoresques :

Ce soir-là, une belle lune ronde s'était levée au-dessus de la forêt, et l'on aurait pu ramasser à sa clarté une pièce de monnaie tombée à terre. Soudain, retentirent allègrement les coups précipités du petit tambour au son grêle. Alors, ce fut dans toutes les paillotes un grand remue-ménage. Les femmes se hâtaient d'éteindre les foyers allumés [...]. Puis s'étant lavé les mains pour ne pas salir leur toilette, elles sortirent de coffres des camisoles fraîches, les pagnes bleus de Saint Louis, et ceux de Bissao et des îles du Sud aux fils de coton multicolores, les mouchoirs de soie ou de madras, que les unes nouaient sans élégance, et que les autres arrangeaient artistement sur leur tête à la manière des grandes coquettes de Fatik ou de Kaolack, les colliers de perles d'or creuses, les bracelets d'or de N'Galam et les verroteries de bazar [...]. A présent, les deux tambours de Bourama Seck et de son fils menaient ensemble leur tapage, l'un grave, l'autre clair, tandis que d'une voix suraiguë le vieux griot chantait la louange de Diouf :

Samba, tu es un homme ! Et le voyage au pays des Toubabs T'a grandi, en vérité... [...] Que tu ailles ou que tu viennes, Tes richesses ne tarissent point. Io ! Samba, tu es le parent du roi ! Que tu ailles ou que tu viennes, Tes richesses ne tarissent point. [...] Ils ont tué un de tes bras, Mais celui qui te reste est plus fort Que les deux des autres hommes. (304-305)

Mais si le lecteur peut penser au mythe du retour de l'enfant prodigue, on ne saurait parler de dénouement heureux. Un cycle de déceptions attend Samba. Il est manchot, ayant perdu un bras à 
la guerre. La rivière a presque englouti le troupeau qui l'attendait comme héritage. Yamina, la fiancée qu'il croyait l'attendre, porte l'enfant d'un autre homme. Dépossédé jusqu'à l'amour auquel il s'accrochait, il se résigne à donner une leçon de morale à la jeune fille : «Depuis ton enfance, Yamina, lui dit-il, en baissant la voix, je cultive les champs de ton père, je lui ai procuré de lourdes récoltes d'arachides et de mil, et j'ai porté tous les jours à ta mère du poisson frais. Tout cela méritait que tu ne m'oublies pas et que je n'aie pas travaillé pour la brousse toute nue » (299300). Samba perçoit clairement cet acte de trahison de la part de sa fiancée comme la violation d'un pacte social. Il ne lui reste que la médaille que les Toubabs lui ont donnée en guise de rétribution : «Ils [l'ennemi allemand] m’ont touché, j'ai crû être mort ; ils m’ont soigné à l'hôpital, ils m’ont donné une médaille » (112). Son usage du pronom «ils » pour désigner allusivement les Toubabs, Allemands et Français à la fois, cache une certaine déception de sa part. Samba a tout perdu (son bras, sa fiancée, son troupeau) pour la France et comme récompense on ne lui a donné qu'une médaille. Ferdinand Oyono abordera plus tard cette mise en scène coloniale autour de la médaille de guerre. Dans son roman classique, Le vieux nègre et la médaille, publié en 1956 chez Julliard, Oyono fait le portrait de Meka, un vieil homme qui a tout donné au colon, y compris ses terres à la mission catholique et ses deux enfants à l'armée coloniale pendant la deuxième guerre mondiale. Ses enfants y meurent pour la France. L'humour dérisoire qui émaille le roman, cache l'amer procès que Oyono fait contre le colon pour son manque de rétribution du service (sacrifice et dévouement) des soldats africains. Ousmane Sembène portera aussi la figure du soldat africain désabusé sur l'écran en 1988. Dans son film Camp de Thiaroye, le cinéaste traite du massacre colonial de tirailleurs sénégalais en 1944 dans un camp au sud de Dakar juste après la guerre. Suite à une rébellion contre leur mauvais traitement et contre le retard de paiement de leur pension de guerre, ces tirailleurs avaient été massacrés par l'armée française. A travers romans et films documentaires, les frères Tharaud, plus tard Oyono et Sembène ouvrent un des chapitres sombres de l'histoire des tirailleurs sénégalais dans les guerres de libération de la France.

La randonnée de Samba Diouf témoigne de l'histoire des tensions entre l'Islam, la colonisation et les sociétés traditionnelles africaines à l'époque coloniale. L'hégémonie de la société traditionnelle africaine reposait sur des structures sociales, politiques et religieuses bien ancrées. La subversion définitive de ces schèmes va s'opérer certes à partir du onzième siècle sous le développement des foyers islamiques, mais surtout au dix-neuvième siècle à cause de l'implantation coloniale. Au-delà de la condition humaine qu'il dépeint dans le contexte de la guerre, $L a$ 
randonnée de Samba Diouf est une restauration de la mémoire et de l'imaginaire africains. Les frères Tharaud substituent subtilement les voix et paroles des colonisés à la voix et parole coloniales. Ainsi réaniment-ils des espaces de performance (le village, la forge, le cosmos, etc.) dans les décors et scènes africains. Ils utilisent des procédés techniques narratifs variés tels le discours direct (dialogues) et des emprunts linguistiques qui participent activement à ce jeu de restitution de la mémoire. Des mots comme «Toubab », « kanou », « sarzent », formes altérées du français, se joignent à l'emploi d'autres vocables du terroir tels « m'boubou », « térés », « m'bar », «sabar ». Le texte est émaillé aussi de proverbes littéralement traduits des langues africaines. Le lecteur découvre ainsi l'imagination et la touche savante des Tharaud. A l'instar de Batouala de René Maran publié en 1921 chez Plon, un an avant La randonnée de Samba Diouf, on peut dire sans aucun doute que les frères Tharaud, et derrière eux Demaison, ont le mérite d'être parmi les précurseurs ou avant-gardistes du projet de décolonisation de l'Afrique occidentale française.

\section{Ouvrages cités}

Becker, Charles. «Les familles paternelles sereer: répartitions par pays traditionnels et par castes ». Bulletin de l'IFAN 44, B. 3-4 (Juillet-Octobre 1982) : 321-410.

Boilat, David. Esquisses Sénégalaises. Paris : Karthala, 1984.

Chevrier, Jacques. Littérature africaine. Histoire et grands thèmes. Paris : Hatier, 1987.

Crétois, Léonce. Dictionnaire sereer-français. Tome 3. Dakar : Clad, 1974.

Deroo, Eric. « La force noire ». Entretien avec Timothy Mirthil. 4 mai 2006.

--- La force noire (2007). Film documentaire (26 mns).

Diop, Birago. Les contes D'Amadou Koumba. Paris : Fasquelle, 1947.

Dupire, Marguerite. Sagesse sereer. Essais sur la pensée sereer ndut. Paris : Karthala, 1994.

Gravrand, Henry. La civilisation sereer: Pangool (Les Esprits). Dakar: Nouvelles Editions Africaines du Sénégal, 1990.

Laye, Camara. L'enfant noir. Paris : Pocket, 1954.

Maran, René. Batouala. Paris : Albin Michel, 1921.

Niane, Djibril Tamsir. Soundjata ou l'épopée Mandingue. Paris : Seuil, 1960.

Oyono, Ferdinand. Le vieux nègre et la médaille. Paris : Julliard, 1956.

Propp, Vladimir. Morphologie du conte. Paris : Seuil, 1965. 
Sartre, Jean Paul. «Orphée Noir », in Senghor Léopold Sédar Anthologie de la nouvelle poésie nègre et malgache de langue française. Paris : PUF, 1948 : 27.

Sène, Papa Massène. «La parole pensée, impératifs et contraintes dans la littérature orale ». Notre Librairie, la littérature sénégalaise 81 (Octobre-Décembre 1985) : 5.

Senghor, Léopold Sédar. Euvre poétique. Paris : Seuil, 1964.

--- Préface. Nouveaux Contes d'Amadou Koumba. Paris : Présence Africaine, 1958.

--- Ethiopiques. Paris : Seuil, 1956.

Tharaud, Jérôme et Jean. La randonnée de Samba Diouf. Paris : Plon, 1922.

\footnotetext{
Notes

${ }^{1}$ L'empire du Manding était constitué de douze provinces. Après les conquêtes de Soundjata Kéita (épelé aussi Keïta), le nombre des provinces s'était considérablement accru, formant ainsi une confédération de plusieurs tribus, y compris la tribu des Kéita, des Kondé, des Traoré, des Camara, et celle des Koroma. Soundjata (1190-1255) était le fondateur de cet empire du Mali dont la capitale était Niani, son village natal situé actuellement en Guinée à la frontière du Mali. Soundjata fut proclamé Mansa (Roi des rois) suite à sa victoire contre Soumaoro Kanté (roi du Sosso) en 1235. Au cours de son intronisation, la Charte du Manden fut proclamé et devint l'une des premières Déclarations des Droits de l'Homme.

${ }^{2}$ Voir Chants d'ombre, « Le retour de l'enfant prodigue », poème IV.

3 «Tann », selon Senghor, est une terre plate que recouvre la mer ou le bras de mer à l'époque des grandes marées.

4 «Bolong », selon Senghor, est un mot d'origine mandingue. C'est un bras de mer ou chenal, bordé de palétuviers le plus souvent.

5 « Kouss », selon Senghor, renvoie aux « Génies qui rappellent les premiers habitants de l'Afrique noire, les Pygmées, qui furent exterminés ou refoulés par les Grands-Nègres » (postface, Ethiopiques)
} 